Purpose This study aimed to examine the impact of introducing or expanding children's community nursing (CCN) services, and understand the contextual mechanisms that mediate the success of planning and implementing such service change. This paper presents findings about the identified contextual mechanisms: the perceived factors that mediated efforts to plan and implement new or expanded CCN services.

Methods A case study design was used with five health communities in England that had recently, or were in the process of, introducing or expanding their CCN provision. Data were collected through twenty-one longitudinal (time frame), in-depth interviews and 6 focus groups with 41 children's community nurses, managers and commissioners. Interviews and focus groups explored the processes, barriers and facilitators to service change planning and implementation. Documentary evidence was also collected. Data were managed using the Framework approach, and analysed thematically.

Results Service change was implemented as planned in three sites, and partially in one site. Intended change was not achieved in one site. Dedicated finance, medical buy-in of CCN, and (where multiple providers were involved) use of a centralised network, all facilitated the achievement of service change. Competition between providers and NHS reforms made service planning complex and time consuming. An absence of medical support for CCN services, and a lack of commitment from commissioners, prevented intended service change in two sites. Communicating service change was deemed important for integration with, and take up by, other local NHS services. However, CCN team staff struggled to do this alongside care delivery and talked of the need for 'marketing'. Demonstrating value and impact of the new and expanded services was challenged by inadequate data collection systems and inaccessible or overly complex data. Staff were keen to explore ways of measuring quality-based outcomes.

Conclusions Issues of visibility and acceptability of CCN services appear to pervade the reported difficulties and successes of planning and implementing service change. Implications for care 'closer to home' policy and practice will be discussed.

\section{G11 TRANSITION FROM HOSPITAL TO HOME: PSYCHOSOCIAL ADAPTATION AND ADJUSTMENT IN PARENTS OF INFANTS WITH SINGLE VENTRICLE HEART CONDITIONS}

${ }^{1} \mathrm{KL}$ Gaskin, ${ }^{2} \mathrm{~S}$ Hutchinson. ${ }^{1}$ Department of Nursing, Midwifery and Paramedic Science, University of Worcester, UK; ${ }^{2}$ Little Hearts Matter, Birmingham, UK

10.1136/archdischild-2015-308599.11

Aims The study presented here retrospectively explored psychosocial adaptation and adjustment in parents going home for the first time with their infants following first stage cardiac surgery for single ventricle heart condition.

Methods Parents, all members of Little Hearts Matter (LHM, a UK Congenital Heart Disease Charity) completed an online questionnaire which asked about family demographics, time of diagnosis, location of specialist heart centre, distance from home, discharge information, social support, parents' feelings about going home, confidence and anxiety at the time of discharge (T0) and at the time of completing the questionnaire (T1). A non-experimental survey design was used; the questionnaire was structured so that both parents could answer the questions independently. 62 families with infants aged between $0-2$ years were sent an email via LHM inviting them to complete the online questionnaire during Nov 2012. There were 22 responses (35\% response rate) from 6 couples, 15 mothers and 1 father. Care had been received at 11 UK specialist cardiac centres and 1 in Australia.

Results There were no statistically significant differences between demographic data (age, education, ethnicity, employment, living arrangements, household income) when compared against mother's anxiety and confidence at T0 or T1; however mothers living $>100$ miles away from the specialist cardiac centre were statistically significantly more anxious at discharge T0 ( $\mathrm{p}=0.025$ ) than those living $<20$ miles away. Mothers whose infant had a hypoplastic left heart were statistically significantly more anxious at discharge than those whose infants had hypoplastic right heart $(\mathrm{p}=0.006)$ as were Fathers with a secondary school education compared to a Bachelor's degree ( $\mathrm{p}=$ $0.031)$ at T0 and T1 ( $p=0.026)$. There was a statistically significant difference when comparing mothers' anxiety levels at discharge T0 and T1 $(\mathrm{p}=<0.0005)$ and confidence scores at T0 and T1 $(\mathrm{p}=<0.0005)$ and Fathers' anxiety levels at discharge $\mathrm{T} 0$ and $\mathrm{T} 1$ ( $\mathrm{p}=<0.018)$.

Conclusion This is the first study of its kind to explore parents' psychosocial adaptation and adjustment during the transition from hospital to home following first stage cardiac surgery for single ventricle condition. The study provides new insights into transition for these parents. Implications for practice will be discussed.

\section{G12 DELIVERING EFFECTIVE NURSING CARE TO CHILDREN AND YOUNG PEOPLE OUTSIDE OF A HOSPITAL SETTING}

${ }^{1} \mathrm{LS}$ Whiting, ${ }^{2} \mathrm{C}$ Caldwell, ${ }^{1} \mathrm{M}$ Donnelly, ${ }^{1} \mathrm{D}$ Martin, ${ }^{1} \mathrm{M}$ Whiting. ${ }^{1}$ Nursing and Social Work, University of Hertfordshire, Hatfield, UK; ${ }^{2}$ Health England North Central and East London LETB, London, UK

\subsection{6/archdischild-2015-308599.12}

"I have found being able to access care at home, or in a non-hospital setting to be invaluable to improving my health." [Young person participant]

This presentation will provide an overview of an exploratory study that was financed and commissioned by Health England North Central and East London Local Education and Training Board [HE NCEL LETB] and was undertaken by the University of Hertfordshire between February and August 2014.

Research question "What education, preparation and development is required to ensure a workforce of nurses who have the requisite knowledge, skills and professional attributes to meet the healthcare service needs of the CYP [Children and Young People] population in the LETB geography?"

The study focussed on the 'outside of hospital' [OOH] environment, although health visiting and school nursing was excluded. Ethical approval was granted by the University of Hertfordshire.

Methods A mixed methods data collection approach was adopted.

Consultation, by questionnaire, with young people $(\mathrm{n}=14)$ via two established Youth Advisory Panels (National Youth Agency; Royal College of Paediatrics and Child Health).

Consultation with nursing staff working in $\mathrm{OOH}$ settings in the NCEL area; data was collected via questionnaires sent to the $11 \mathrm{CCN}$ teams (response rate: 64\%), and, via individual semistructured interviews with clinical nurses

Consultation with four Higher Education Institute [HEI] providers within the NCEL area; semi-structured interviews with 